\title{
Progressive Decline in Hippocampal CAI Volume in Individuals at Ultra-High-Risk for Psychosis Who Do Not Remit: Findings from the Longitudinal Youth at Risk Study
}

\author{
New Fei Ho*,1,2, Daphne J Holt ${ }^{3}$, Mike Cheung ${ }^{4}$, Juan Eugenio Iglesias', Alex Goh', Mingyuan Wang', \\ Joseph KW Lim², Joshua de Souza ${ }^{2}$, Joann S Poh ${ }^{2}$, Yuen Mei See', Alison R Adcock ${ }^{6}$, Stephen J Wood', \\ Michael WL Chee ${ }^{2}$, Jimmy Lee ${ }^{1,8}$ and Juan Zhou ${ }^{*, 2,8}$ \\ IInstitute of Mental Health, Singapore, Singapore; ${ }^{2}$ Neuroscience and Behavioral Disorders Program, Center for Cognitive Neuroscience, \\ Duke-National University of Singapore Graduate Medical School, Singapore, Singapore; ${ }^{3}$ Department of Psychiatry, Massachusetts General \\ Hospital and Harvard Medical School, Boston, MA, USA; ${ }^{4}$ Department of Psychology, National University of Singapore, Singapore, Singapore; \\ ${ }^{5}$ University College London, London, UK; ${ }^{6}$ Center for Cognitive Neuroscience, Duke University, Durham, NC, USA; ${ }^{7}$ School of Psychology, University \\ of Birmingham, Birmingham, UK
}

\begin{abstract}
Most individuals identified as ultra-high-risk (UHR) for psychosis do not develop frank psychosis. They continue to exhibit subthreshold symptoms, or go on to fully remit. Prior work has shown that the volume of CAI, a subfield of the hippocampus, is selectively reduced in the early stages of schizophrenia. Here we aimed to determine whether patterns of volume change of CAI are different in UHR individuals who do or do not achieve symptomatic remission. Structural MRI scans were acquired at baseline and at I-2 follow-up time points (at 12-month intervals) from 147 UHR and healthy control subjects. An automated method (based on an ex vivo atlas of ultra-highresolution hippocampal tissue) was used to delineate the hippocampal subfields. Over time, a greater decline in bilateral CAI subfield volumes was found in the subgroup of UHR subjects whose subthreshold symptoms persisted $(n=40)$ and also those who developed clinical psychosis $(n=12)$, compared with UHR subjects who remitted $(n=4 I)$ and healthy controls $(n=54)$. No baseline differences in volumes of the overall hippocampus or its subfields were found among the groups. Moreover, the rate of volume decline of CAI, but not of other hippocampal subfields, in the non-remitters was associated with increasing symptom severity over time. Thus, these findings indicate that there is deterioration of CAI volume in persistently symptomatic UHR individuals in proportion to symptomatic progression. Neuropsychopharmacology (2017) 42, I36 I-1370; doi: I0.1038/npp.2017.5; published online I February 2017
\end{abstract}

\section{INTRODUCTION}

Individuals at risk for psychosis are generally identified by the presence of subthreshold symptoms. Many studies of this population largely focus on examining characteristics of at-risk subjects who subsequently develop full-blown psychotic symptoms. Yet, the majority of the at-risk individuals do not convert to frank psychosis (Simon et al, 2011). The subthreshold symptoms observed at baseline typically either persist or resolve over time. It has been argued that clinical care for individuals with persistent subthreshold psychotic

*Correspondence: Dr NF Ho, Institute of Mental Health, I0 Buangkok View, Buangkok Green Medical Park, Singapore 539747, Singapore, Tel: +65 63893819, Fax: +65 63437962, E-mail: new_fei_ho@imh.com.sg or Dr J Zhou, Neuroscience and Behavioral Disorders Program, Center for Cognitive Neuroscience, Duke-National University of Singapore Graduate Medical School, 8 College Road, No. 06-15, Singapore 169857, Singapore, Tel: +65 66012392, Fax: +65 62218625, E-mail: helen.zhou@duke-nus.edu.sg

${ }^{8}$ Joint senior authors.

Received 29 September 2016; revised 5 December 2016; accepted 4 January 2017; accepted article preview online 12 January 2017 symptoms is indicated, given the functional impairment seen in this population (Carpenter, 2014). To stratify treatment for at-risk individuals with varying outcomes (Kapur et al, 2012), attention needs to be directed towards understanding the biological predictors of these potential outcomes, as it has become increasingly clear that in psychiatric illnesses, biological changes precede the appearance of symptoms in a dimensional manner (Cuthbert and Insel, 2010).

Several lines of evidence suggest that abnormalities of the hippocampus may represent candidate biological markers of psychosis (Heckers and Konradi, 2010). Two recent independent large-scale consortium studies have separately found the hippocampus to exhibit the most extensive volume reductions among all of the subcortical brain regions examined in schizophrenia (Okada et al, 2016; van Erp et al, 2015). Also, several meta-analyses have consistently found hippocampal volume reductions not just in chronic schizophrenia but also within first-episode populations (Adriano et al, 2012; Haijma et al, 2013). However, the timing of the emergence of hippocampal abnormalities during the course of the illness remains unclear. 
Findings from cross-sectional studies of hippocampal volume in at-risk subjects, which have included familial (first-degree relatives) and/or clinical (those with subsyndromal symptoms) at-risk groups, have been mixed thus far-with findings of volume reduction, or no changes (Ganzola et al, 2014; Hurlemann et al, 2008; McDonald et al, 2006; O'Driscoll et al, 2001; Ongur et al, 2006; Seidman et al, 2002; Witthaus, 2010; Witthaus et al, 2009; Wood et al, 2005, 2010). Longitudinal studies of hippocampal volumes in atrisk subjects could help to resolve the discrepancies among prior findings by determining whether volume deficits of the hippocampus are already present during the prodromal phase of psychosis, and map the potential differences in the trajectories of hippocampal volumes for subjects with different outcomes.

Also, studies must now account for evidence that the cytoarchitecturally distinct subfields of the hippocampus (ie, CA1, CA2/3, CA4, dentate gyrus, and subiculum) are differentially affected by the disease process associated with psychosis (Talati et al, 2014), particularly early on in the illness (Ho et al, 2016; Schobel et al, 2013). Metabolic abnormalities selectively in the CA1 subfield of the hippocampus have been found to precede the development of psychosis and a subsequent change in the shape of a hippocampal region likely corresponding to CA1 (Schobel et al, 2013). Consistent with these findings, we recently identified, using a validated automated method of segmenting the hippocampus (Iglesias et al, 2015), a selective volume deficit in the CA1 subfield in early-course schizophrenia patients (Ho et al, 2016). Therefore, in the current investigation, we sought to extend this finding by determining whether CA1 volume deficits are present during the prodrome and are predictive of persistence or worsening of symptoms.

To test this hypothesis, we prospectively followed a group of ultra-high-risk (UHR) individuals, who met the consensus criteria for the UHR state (Yung et al, 2005), and healthy control (HC) subjects, who reside in a region of the world with one of the lowest rates of illicit substance use worldwide (Lee et al, 2013) and thus are likely minimally affected by the potentially confounding effects of substance use on hippocampal volume (Solowij et al, 2013; Thompson et al, 2004). We collected MRI scans and clinical measures at the time of recruitment (baseline), and at the 12- and 24-month followup time points, or until the point of transition to clinical psychosis. Based on prior work (Ho et al, 2016; Schobel et al, 2013), we predicted that we would observe a progressive decline in volume of the CA1 subfield in the subgroup of UHR subjects who exhibited persistent or worsening symptoms (the 'non-remitters') but this decline would be absent in the UHR subjects who remitted.

\section{MATERIALS AND METHODS}

\section{Participants}

We studied 93 UHR individuals and 54 HCs. These participants were part of the Longitudinal Youth At-Risk Study conducted from 2008 to the fall of 2015 in Singapore (Lee et al, 2013; Wang et al, 2016). The UHR participants met the criteria for the at-risk mental state defined by the Comprehensive Assessment of At-Risk Mental States
(CAARMS) version 12/2006 (Yung et al, 2006), which includes the positive subscale of the CAARMS and the Social and Occupational Functioning Assessment Scale (SOFAS) (Goldman et al, 1992; Yung et al, 2005); the youths were of genetic risk for psychosis and/or showed subthreshold symptoms, and deterioration in functioning in the past 12 months. See Supplementary Methods S1 for details of the recruitment and screening process.

Exclusion criteria for the UHR participants included (i) past or current history of psychosis, or mental retardation, (ii) any treatment with mood stabilizers or antipsychotic medications, (iii) medical conditions that could account for their prodromal psychotic symptoms, (iv) current substance use, abuse, or dependence, and ( $\mathrm{v}$ ) current alcohol abuse or dependence, assessed using the Structured Clinical Interview for DSM-IV Axis I Disorders-Patient version (SCID-I/P) (First, 2002b).

Exclusion criteria for the HC included (i) a history of major psychiatric conditions, (ii) first-degree relatives with any axis I disorder, and (iii) any history of substance or alcohol abuse/dependence, assessed using the Structured Clinical Interview for DSM-IV Axis I Disorders-NonPatient version (SCID-I/NP) (First MB, 2002a). In addition, the HC did not meet the CAARMS criteria for UHR status.

General exclusion criteria for all of the subjects included contraindications for MRI scans, and major medical conditions. Ethics approval for this study was provided by the Singapore National Healthcare Group's Domain Specific Review Board. Written informed consent was obtained from all participants aged 21 years and above, or from a legally acceptable representative for participants under 21 years of age with their assent.

\section{Clinical Measures}

The CAARMS and SOFAS, as part of the CAARMS version $12 / 2006$, were administered to the UHR subjects at 6-month intervals, or until the point of transition to clinical psychosis. The SCID-I/P was administered every 12 months to the UHR subjects. The SCID-I/NP was administered to the HC subjects during the baseline visit and at the end of followup, which was 24 months later.

The UHR subjects were assigned to three different groups, based on their combined CAARMS and SOFAS status at their last MRI scan. UHR subjects were deemed to have remitted if they no longer met the CAARMS/SOFAS criteria for UHR status (UHR remitters, UHR-R). UHR subjects who transitioned to psychosis during the course of study, and UHR subjects who remained at risk because of persistent prodromal symptoms were combined into one group to afford greater power for subsequent analyses (UHR-nonremitted, UHR-NR). Two of the UHR subjects with unstable symptomatology, that is, who were symptom free for 12 months, and then experienced a second onset of subsyndromal symptoms, were excluded from the primary analysis.

\section{MRI Acquisition and Image Processing}

Parameters of the structural scans, which were performed at baseline, 12 months, and 24 months, are detailed in Supplementary Methods S2. The MRI scans were performed 
within 2 weeks after the clinical and neuropsychological assessments were conducted.

Standard preprocessing of the structural images, followed by longitudinal preprocessing steps that further reduced withinsubject variability, were performed using FreeSurfer version 5.3 (http://surfer.nmr.mgh.harvard.edu) (Fischl et al, 2002, 2004; Reuter et al, 2012). The segmentation of the hippocampus into its subfields was performed using a recently validated segmentation algorithm that was based on an atlas constructed from ultra-high-resolution ex vivo hippocampal tissue (Iglesias et al, 2015). Details of these methods can be found in Ho et al (2016). Visual quality control of the image processing that included checking for excessive motion was performed (Supplementary Methods S3). The proportion of UHR subjects excluded because of motion was not different from healthy subjects. Further, data set from an UHR-NR subject was removed from the analysis because of poor longitudinal image reconstruction. Hippocampal segmentation results were visually checked for each subject; there were no instances of incomplete labeling of hippocampal subregions or mislabeling of extrahippocampal regions in the remaining 147 subjects.

Volumes of the seven subfields of the hippocampus (Burwell and Agster, 2009): the GCL, CA4, CA2/3, CA1, $\mathrm{ML}$, the hippocampal tail, and the subiculum were extracted. The volumes of the seven subfields for each hemisphere were summed to obtain a measure of the volume of the global left and global right hippocampus. To facilitate comparisons with other studies, the volumes of the hippocampus (from the standard output of the FreeSurfer automated labeling of major subcortical structures) were also extracted for a supplementary analysis.

\section{Statistical Analyses}

All analyses were performed using open-source R software (version 3.2) (The R Core Team, 2015).

Participant characteristics. We examined three groups of participants: HC, UHR-R, and UHR-NR. Baseline betweengroup demographic, clinical, and cognitive differences were tested using $\chi^{2}$ tests and analysis of variance (ANOVA).

Primary analyses. We tested whether there was a main effect of group on baseline volumes, as well as an interaction effect between group and time, by fitting a linear mixedeffects model for each volume measure. Before model fitting, Shapiro-Wilks test for normality and the Bartletts test of homogeneity of variance were first performed to test for normal distributions and equal variances of all the volume measures of the hippocampus and subfields at each time point among the groups.

In our model, the fixed-effects included group, time (in months), interaction between group and time, ICV, baseline age, and gender. Random effects included individual intercept and individual slope of time. An autoregressive structure was used to model the variance-covariance matrix, which recognizes that proximal observations are more correlated than distant observations. An ANOVA was performed on the linear mixed-effects model to test whether there was statistical evidence of group differences in intercept (effect of group at baseline) and slope (interaction effect between group and time). If so, this was followed by post hoc pair-wise group comparisons using Wald tests to compute asymptotic $\chi^{2}$ statistics, with $p$-values adjusted for betweengroup multiple comparisons using the Holm-Bonferroni method (Fox and Weisberg, 2011; Hothorn et al, 2008).

Secondary analyses. (1) We first tested whether the presence of psychiatric comorbidity (ie, SCID-assessed lifetime history of depressive spectrum disorders, anxiety spectrum disorders, alcohol abuse/dependence, and/or substance abuse/dependence) influenced our primary hypotheses by adjusting for comorbidity in the statistical model. (2) We also tested whether treatment with antidepressants or benzodiazepines at any time-point affected the findings by adding these as time-varying predictors in the model. (3) We then tested whether the inclusion of two subjects with fluctuating symptoms over time impacted the primary findings. (4) Last, we tested whether CA1 volume decline was different in individuals who developed clinical psychosis, by further subtyping the non-remitted UHR subjects into those who remained symptomatic at a subthreshold level (UHR-NR-non-psychosis or 'non-converters') and those who transitioned to full psychosis (UHR-NR-psychosis or 'converters').

Post hoc correlations between symptoms and hippocampal subfields found to exhibit group differences. We examined the within-group relationship between rates of volume change in the affected hippocampal subfields and symptoms. First, we fitted linear mixed-effect models for symptom measures, with CAARMS scores as dependent variables; time, age, and gender as fixed-effect variables; and individual intercept and slope of time as random-effect variables. The slope estimates of the subjects' measures of symptoms were then regressed onto the slope estimates of the affected hippocampal subfield volumes (extracted from the linear mixed-effect models constructed for the above-mentioned primary hypothesis).

\section{RESULTS}

\section{Subject Cohort Characteristics at Baseline and Follow- Up}

At baseline, the three groups of subjects, the HC $(n=54)$, UHR-R $(n=41)$, and UHR-NR $(n=52$, including 12 whose symptoms worsened to exceed subthreshold), were well matched in terms of age, gender, ethnicity, handedness, and ICV (which was used as an estimate of head size) (Table 1). A further breakdown of the baseline clinical characteristics of the UHR subpopulations, that is, a comparison of the UHR$\mathrm{R}$ and UHR-NR, found no group differences in CAARMS scores, as well as the proportion of individuals with (1) lifetime and/or current history of depressive or anxiety disorder, (2) past history of alcohol abuse or dependence, and/or (3) past history of substance abuse or dependence (Supplementary Table S1). 
Table I Baseline Sociodemographic, Imaging, and Clinical Characteristics of Three Groups Healthy Controls, UHR for Psychosis subjects who Subsequently Remitted (UHR-R), and the Combined Group of UHR Subjects who Remained at Risk/Converted to Psychosis (UHR-NR)

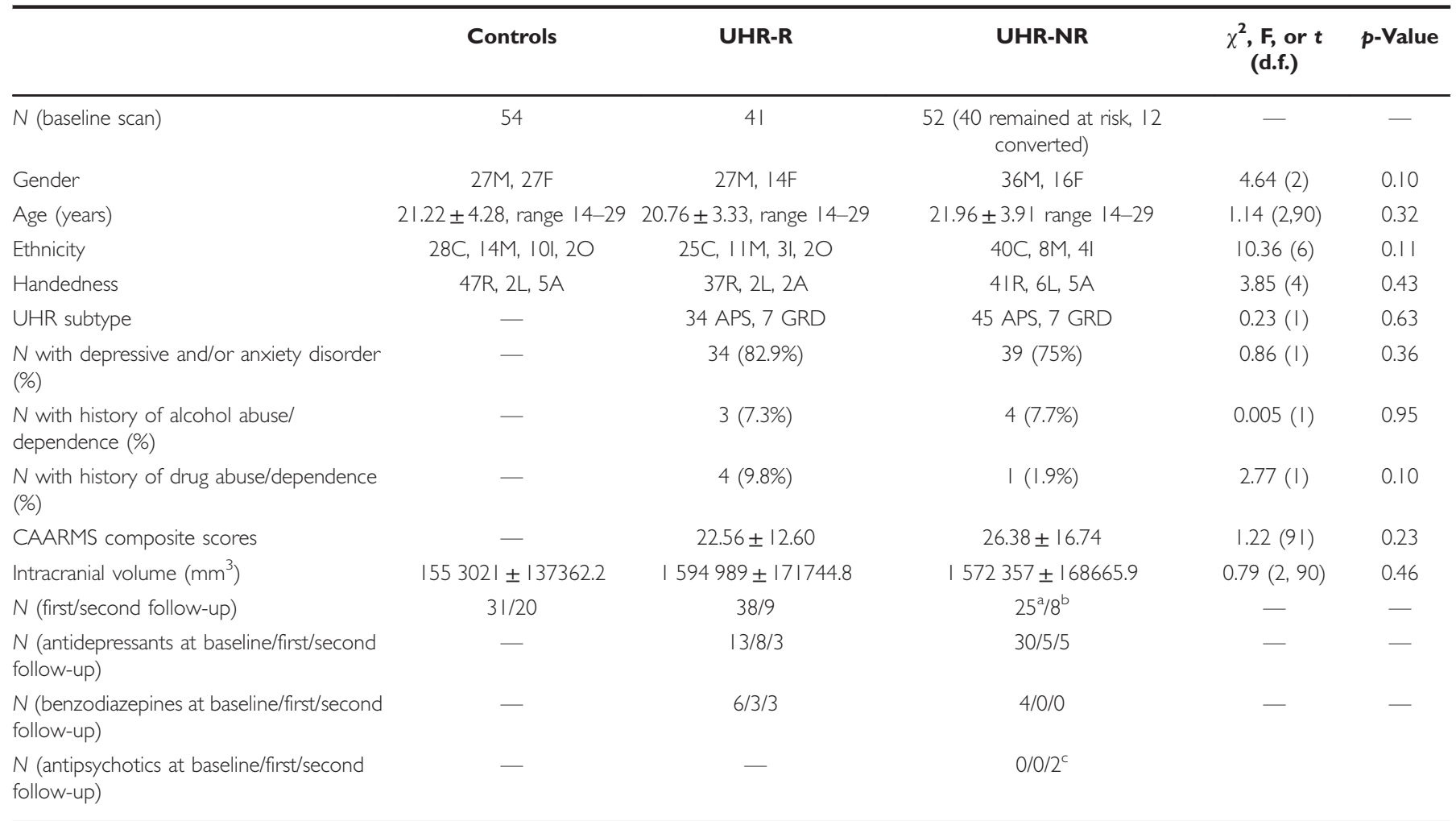

Abbreviations: A, ambidextrous; APS, attenuated psychotic syndrome; C, Chinese; CAARMS, Comprehensive Assessment of At-Risk Mental States; d.f., degrees of freedom; F, females; I, Indian; L, left; $M$, males; $M$, Malay; MRI, magnetic resonance imaging; $O$, others; $R$, right; UHR, ultra-high-risk; $V$, vulnerability/genetic risk group. Measures of continuous variables are indicated by their means \pm standard deviation. $\chi^{2}$, analysis of variance, or independent $t$-tests were used to examine possible group differences in the variables.

aWe conducted the first follow-up MRI scan for 5 of the UHR subjects who had developed psychosis, but did not conduct the second follow-up scan for these five subjects.

${ }^{b}$ Among the subjects with second follow-up scans, one subject had converted to psychosis between the first and second follow-up, whereas one subject had converted to psychosis 10 months after the second follow-up scan.

${ }^{c}$ At the point of the second follow-up, two participants were newly diagnosed with clinical psychosis and were prescribed antipsychotics (risperidone and quetiapine). The others were scanned before receiving medication.

\section{No Group Differences in Hippocampal Volumes at Baseline}

At baseline, no between-group differences in global measures of bilateral hippocampal volumes were found; also, similar results were found using the standard subcortical segmentation method (see Supplementary Table S2). However, a significant group by time effect was found for the volume of the right (but not the left) global hippocampus $\left(F_{2,148}=9.69\right.$, $p=0.0001$, Table 2A). Post hoc analyses found that UHR-NR, compared with UHR-R and HC subjects, showed greater decline in right hippocampal volume over time (HC and UHR-NR groups: $\beta=3.7, \chi^{2}=14.5, p<0.0003$; UHR-R and UHR-NR group: $\beta=3.93, \chi^{2}=17.8, p<0.0001$ ) (Figure 1a).

\section{Progressive Volume Decline of the CA1 Subfield in Non- Remitting UHR Subjects}

We then tested our primary hypothesis that a volume decline in CA1 would be observed over time in UHR-NR, compared with UHR-R subjects. While no initial group differences were found in the volume of CA1 in both the left and right hemispheres, a significant group by time interaction effect was observed (right: $F_{2},{ }_{148}=9.115, \quad p=0.0002$; left: $\mathrm{F}_{2,} \quad 148=3.077, p=0.049$; Table $\left.2 \mathrm{~B}\right)$. Post hoc analysis (adjusted for multiple comparisons between groups) revealed that the CA1 volume decline was more pronounced in the UHR-NR compared with HC (right: $\beta=1.05, \chi^{2}=7.33$, $p<0.013$; left: $\left.\beta=0.9, \chi^{2}=5.74, p=0.049\right)$ and UHR-R subjects (right: $\beta=1.59, \chi^{2}=18.15, p<0.0001$; left: $\beta=0.77$, $\chi^{2}=4.42, p=0.07$ ), but there was no such difference between the UHR-NR and HC subjects (Figures $1 \mathrm{~b}$ and $\mathrm{c}$ ). These findings remained unchanged after adjusting for comorbidity (which had an effect on several other subfields but not CA1; see Supplementary Table S3) and treatment with benzodiazepine and antidepressant medication (Supplementary Table S4A). Also, inclusion of two subjects with fluctuating symptomatology (in a secondary analysis) did not substantively alter the results (Supplementary Table S5).

\section{No CA1 Volume Trajectory Differences Between Non- Remitting Converters and Non-Converters}

Secondary analyses of additional subgroups (UHR-R, UHRNR-non-psychosis, and UHR-NR-psychosis) and HC 
Table 2 Summary Statistics of Changes in Volumes of The Hippocampus and its Subfields Over Time in HC Subjects, UHR-R Subjects, and UHR-NR Subjects (Those who had Persistent Subthreshold Symptoms and Those who Subsequently Developed Clinical Psychosis)

\begin{tabular}{|c|c|c|c|c|}
\hline & \multirow{2}{*}{$\begin{array}{c}\text { ANOVA of group by } \\
\text { time }\end{array}$} & \multicolumn{3}{|c|}{ Post hoc statistics of group by time effect } \\
\hline & & UHR-R and HC & UHR-NR and HC & UHR-NR and UHR-R \\
\hline Left & $F_{2,148}=0497, p=0.61$ & & & \\
\hline Right & $F_{2,148}=8.18, p=0.0004^{a}$ & $\begin{array}{c}\beta=0.2, \chi^{2}=0.09, p=0.76 \\
C l=-1.60,0.48\end{array}$ & $\begin{array}{c}\beta=-2.94, \chi^{2}=12.09, p=0.0007 \\
C l=-4.45,-1.17\end{array}$ & $\begin{array}{c}\beta=-3.14, \chi^{2}=15.06, p=0.0002 \\
C l=-1.60,0.49\end{array}$ \\
\hline \multicolumn{5}{|l|}{ (B) $C A I$} \\
\hline Left CAI & $F_{2,148}=3.10, p=0.048^{a}$ & $\begin{array}{c}\beta=-0.14, \chi^{2}=0.23, p=0.63 \\
C l=-0.74,0.45\end{array}$ & $\begin{array}{c}\beta=-0.9, \chi^{2}=5.56, p=0.05 \\
C l=-0.16,-0.15\end{array}$ & $\begin{array}{c}\beta=-0.76, \chi^{2}=4.26, p=0.07 \\
C l=-1.46,-0.02\end{array}$ \\
\hline Right CAI & $F_{2,148}=9.08, p=0.0002^{a}$ & $\begin{array}{c}\beta=0.54, \chi^{2}=2.93, p=0.09 \\
C l=-0.08,1.16\end{array}$ & $\begin{array}{c}\beta=-1.05, \chi^{2}=7.32, p=0.007 \\
C l=-1.8,-0.28\end{array}$ & $\begin{array}{c}\beta=-1.6, \chi^{2}=\mid 8.15, p<0.0001 \\
C l=-2.33,-0.85\end{array}$ \\
\hline Left CA4 & $F_{2,148}=0.62, p=0.54$ & & & \\
\hline Right CA4 & $F_{2,148}=1.24, p=0.30$ & & & \\
\hline Left CA3 & $F_{2,148}=0.83, p=0.43$ & & & \\
\hline Right CA3 & $F_{2,148}=4.97, p=0.008^{a}$ & $\begin{array}{c}\beta=0.09, \chi^{2}=0.56, p=0.46 \\
C l=-0.15,0.33\end{array}$ & $\begin{array}{c}\beta=-0.37, \chi^{2}=5.84, p=0.031 \\
C l=-0.68,-0.068\end{array}$ & $\begin{array}{c}\beta=-0.46, \chi^{2}=9.78, p=0.005 \\
C I=-0.76,-0.17\end{array}$ \\
\hline $\begin{array}{l}\text { Left molecular } \\
\text { layer }\end{array}$ & $F_{2,148}=0.76, p=0.47$ & & & \\
\hline $\begin{array}{l}\text { Right molecular } \\
\text { layer }\end{array}$ & $F_{2,148}=2.65, p=0.07$ & & & \\
\hline Left subiculum & $F_{2,148}=0.91, p=0.40$ & & & \\
\hline
\end{tabular}

Abbreviations: ANOVA, analysis of variance; $\mathrm{Cl}$, confidence interval; $\mathrm{HC}$, healthy control; UHR, ultra-high-risk; UHR-NR, non-remitted UHR; UHR-R, remitted UHR. A linear mixed-effects model was used to test whether there were group differences in volume measures over time. As there were three groups, an ANOVA was first performed to test whether there was statistical evidence of a group by time effect on the following measures: (A) the global hippocampus, that is, the sum of the seven subfields of the hippocampus in the left and right hemisphere of the brain, (B) a priori CAI hippocampal subfields; and (C) the other subfields of the hippocampus. aSignificance at a threshold of $p<0.05$. Post hoc pair-wise group comparisons were then performed using Wald tests (based on sampling covariance matrix of the parameter estimates) to compute an asymptotic $\chi^{2}$, with $p$-values were adjusted for multiple comparisons between the groups using the Holm-Bonferroni method. The $\beta s$ indicate the differences in slopes between the two groups. For example, in the case of right CAI, the slope of UHR-NR relative to HC is - I.05 per month. The $\beta$ s can be considered as unstandardized effect sizes. The 95\% Cls are also shown; a Cl covering the value of zero means the null hypothesis cannot be rejected.

revealed significant group differences in the right CA1 volume trajectory (right: $\mathrm{F}_{3}, 147=6.9, p=0.0002$ ), while group differences in the left CA1 were subtle $\left(F_{3,147}=2.36\right.$, $p=0.074)$. Consistent with the findings of the primary analysis, post hoc tests showed a steeper right CA1 volume decline in the UHR-NR-non-psychosis group compared with the UHR-R $\left(\beta=1.4, \chi^{2}=11.9, p=0.003\right)$, and also in the UHR-NR-psychosis group compared with the HC group $\left(\beta=1.93, \chi^{2}=7.1, p=0.03\right)$ and UHR-R group $(\beta=2.46$, $\left.\chi^{2}=11.9, p=0.003\right)$. The volume trajectories did not differ between the UHR-NR-psychosis and UHR-NR-nonpsychosis groups.

\section{Volume Trajectories of Other Hippocampal Subfields}

With the exception of the right CA3 $\left(\mathrm{F}_{2},{ }_{148}=4.97\right.$, $p=0.0082$ ), the group by time effect was not observed in the other subfields (Table 2C). UHR-NR subjects displayed a steeper volume decline compared with $\mathrm{HC}(\beta=-0.37$, $\left.\chi^{2}=5.84, p=0.03\right)$ and UHR-R subjects $\left(\beta=-0.46, \chi^{2}=9.8\right.$, $p=0.005)$ for the right CA3. However, when we accounted for treatment with antidepressant and benzodiazepine medications, there was no longer a significant group by time effect for the right CA3 (Supplementary Table S4B). Also, the secondary pair-wise comparisons among the further subtyped UHR subjects and HC groups did not reveal any significant group differences in CA3 volumes.

\section{Rate of CA1 Volume Decline Correlates With Rate of Worsening CAARMS Scores}

We then examined whether there was a longitudinal relationship between decline in volume of the affected subfields, that is, right CA1, left CA1, and right CA3, and 


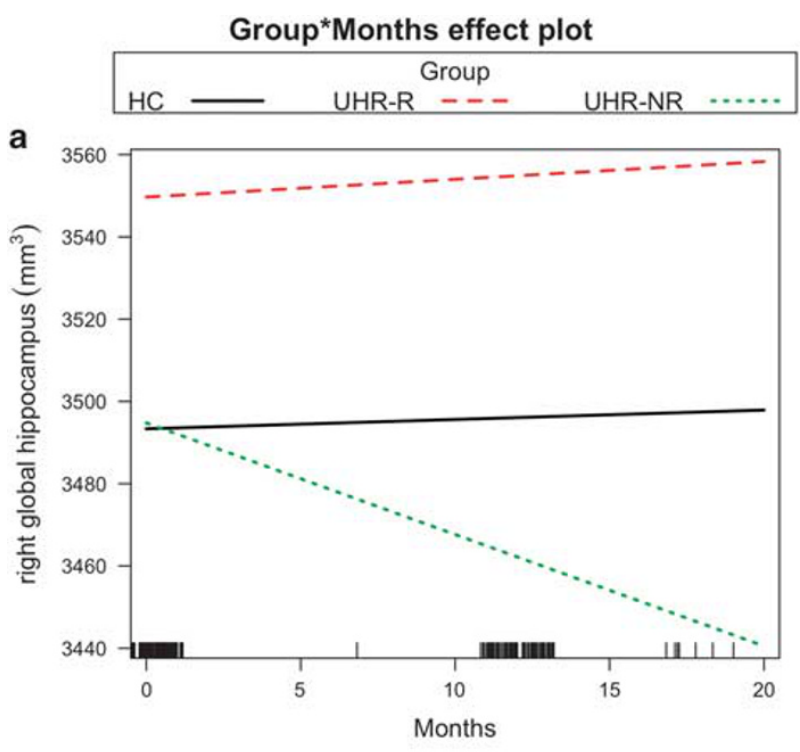

b
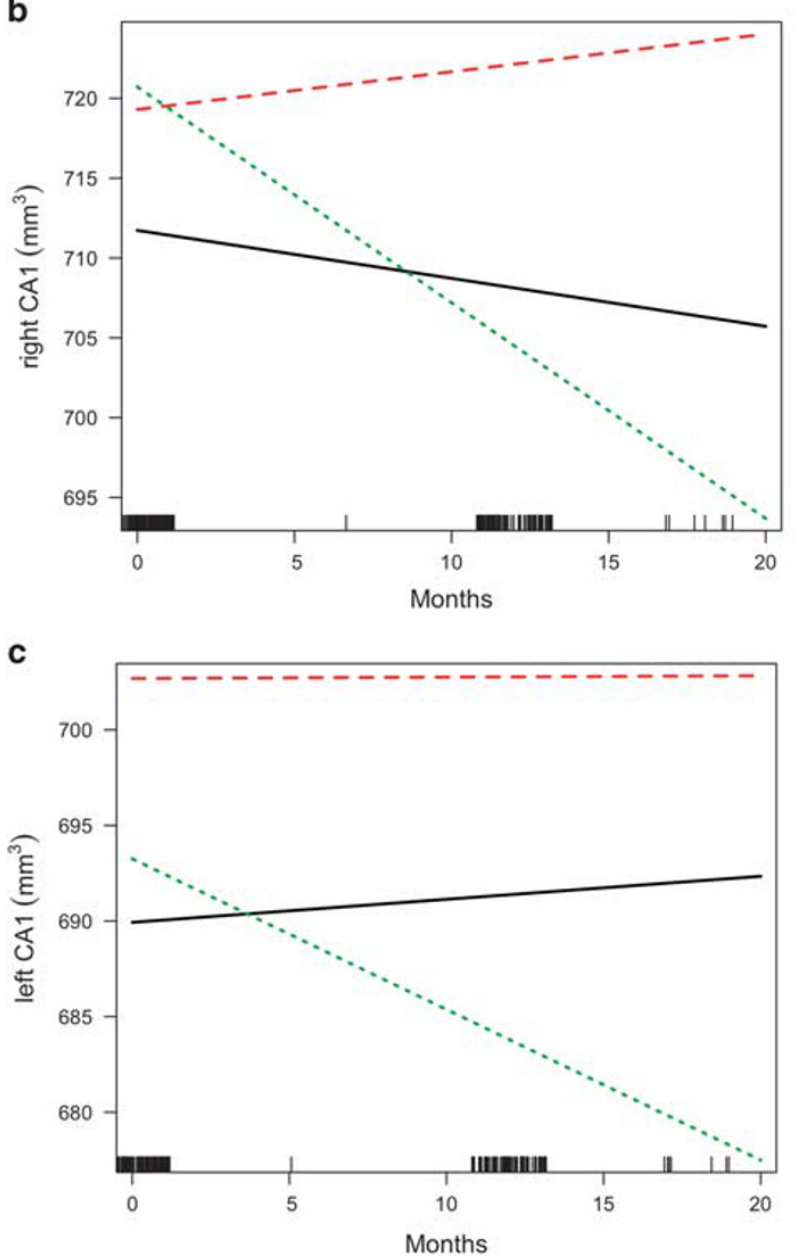

Figure I CAI volume declines over time in ultra-high-risk (UHR) subjects showing persistent or worsening symptoms. Compared with healthy controls (HCs) and UHR subjects who remitted (UHR-R), the UHR 'non-remitters', whose symptoms persisted or worsened over time (UHR$\mathrm{NR}$ ), showed steeper progressive decline in volume in measures of the (a) right hippocampus, that is, sum of hippocampal subfields in the right hemisphere, (b) right CAI, and (c) left CAI. The bars at the bottom of the $x$ axis represent the number of observations over time.
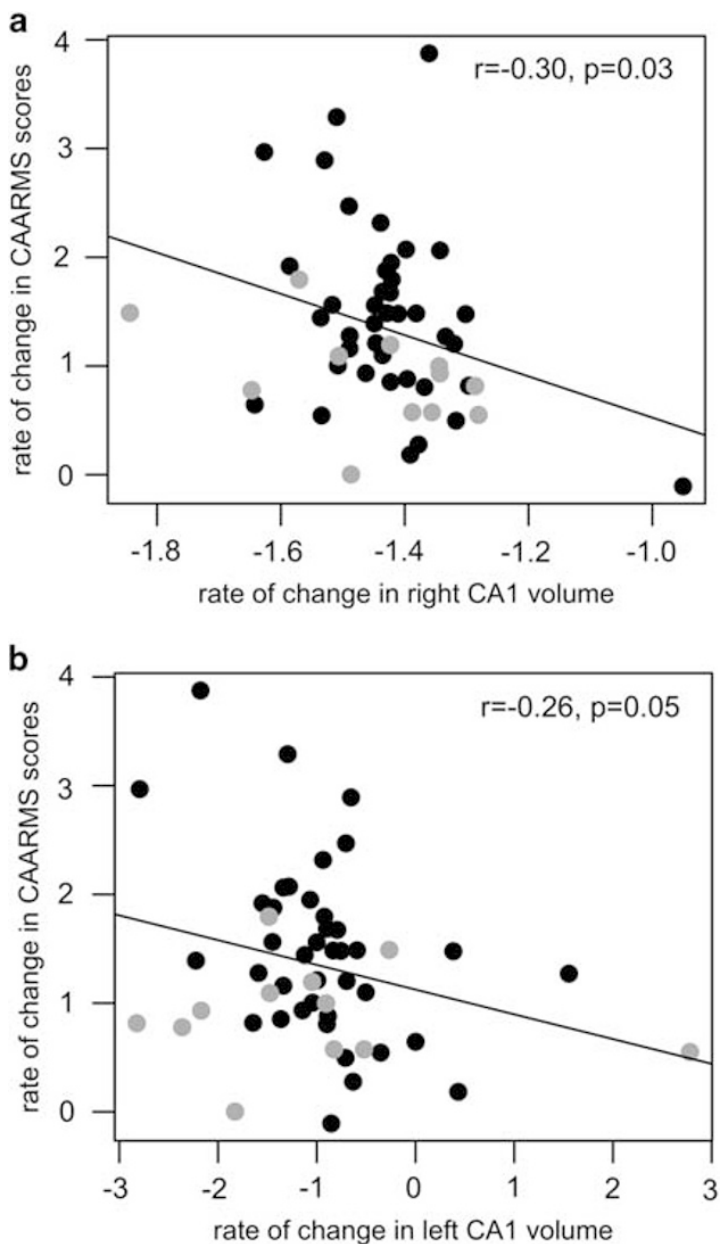

Figure 2 CAI volume decline is associated with worsening of symptoms over time. In the ultra-high-risk non-remitters (UHR-NR), an inverse correlation was found between the rate of change in Comprehensive Assessment of At-Risk Mental States (CAARMS) scores and the (a) rate of change in right $C A I$ volume and (b) rate of change in left CAI volume. Black circles indicate UHR-NR subjects who have persistent but subthreshold symptoms (UHR-NR-non-psychosis); open circles indicate UHR subjects who developed clinical psychosis (UHR-NR-psychosis). Secondary analyses revealed no differences in these CAI volume-symptom relationships between the UHR-NR-non-psychosis and UHR-R-psychosis groups.

change in symptom scores in the UHR-NR group. We found an association between the rates of volume decline of CA1 and rates of increasing severity in CAARMS composite scores (right CA1: $r=-0.30, p=0.03$; Figure 2a; left CA1: $r=-0.26, p=0.05$; Figure $2 \mathrm{~b}$ ). This relationship was not found for the right CA3 volumes. Also see Supplementary Figure S1.

\section{DISCUSSION}

\section{Summary of Main Findings}

At baseline, no volume deficits of the hippocampus or of any of its subfields were observed in a cohort of UHR young adults. Over time, however, progressive decline of the volume of the CA1 subfield was found in UHR subjects who remained persistently symptomatic or developed fullblown psychosis. Moreover, the decline in CA1 volume in this non-remitting group was associated with increasing 
symptom severity. In contrast, CA1 volume remained unchanged in UHR individuals who remitted.

\section{No Initial Hippocampus Volume Deficit in At-Risk Individuals}

The absence of baseline global hippocampal volume deficits in UHR subjects observed here agrees with findings of some prior studies (Buehlmann et al, 2010; Cannon et al, 2015; Klauser et al, 2015; Schobel et al, 2013; Velakoulis et al, 2006) but not others (Dean et al, 2015; Francis et al, 2013; O'Driscoll et al, 2001; Seidman et al, 2002, 2014; Wood et al, 2010). The inconsistency of results of prior studies may be related to variability across studies in the phases at which the UHR subjects were studied, for example, some of the studies may have captured subjects during the late prodrome (when hippocampal changes may have begun or at least become detectable at a macroscopic level), while others may have assessed subjects in early prodromal stages (before measurable changes in hippocampal volumes have occurred) (Addington and Heinssen, 2012; Fusar-Poli et al, 2014). The discrepancies in this literature may also be related to methodologic differences across studies and/or heterogeneity within and across cohorts, that is, variability in MRI acquisition parameters (Bois et al, 2014; O'Driscoll et al, 2001; Seidman et al, 2002) and hippocampal analyses methods used (Bois et al, 2014; Dean et al, 2015; Fusar-Poli et al, 2011), as well as the potentially confounding effects of comorbid mood, anxiety, and substance abuse disorders that have been linked to alterations in hippocampal structure and function (Cha et al, 2016; Smith et al, 2015; Whittle et al, 2014). Of note, the UHR subjects in the present study cohort were never treated with antipsychotic and mood-stabilizing medications, and predominantly lacked histories of substance abuse.

\section{Emergence of CA1 Volume Decline in At-Risk Individuals Differentiates Non-Remitters From Remitters}

Gradual volume decline in bilateral CA1 and right CA3-but not in other hippocampal subfields-was found in subjects who remained persistently symptomatic at a subthreshold level, or whose symptoms worsened to the point of meeting criteria for an episode of clinical psychosis. The trajectory of CA1 volume changes observed here in non-remitting UHR subjects is similar to the pattern of hypermetabolism, which preceded shape abnormalities, of CA1 that was previously found in clinical high-risk subjects who transitioned to psychosis (Schobel et al, 2013). In a recent study, we reported a selective CA1 volume deficit in subjects in the early stages of schizophrenia (Ho et al, 2016). The current observation of a similar pattern of findings in UHR subjects further indicates that changes in the CA1 subfield of the hippocampus occur early on in the illness process and are not attributable to secondary effects of having the illness, such as treatment or chronicity. In addition, the finding of an association between progressive CA1 volume decline in the UHR-NR subjects and increasing symptom severity replicates a similar association observed in patients with schizophrenia (Ho et al, 2016). Taken together, these findings provide converging evidence that CA1 atrophy over time, regardless of illness stage, is closely linked to clinical deterioration.
As the transition to frank psychosis is clinically defined, it is unclear whether criteria for a psychosis diagnosis can be precisely demarcated by any biomarker threshold (Yung et al, 2010). Recently, converging clinical, cognitive, biological, and genetic evidence across psychotic spectrum disorders, and at both early and chronic stages, has supported a dimensional model of psychosis (Craddock et al, 2009; Hill et al, 2013; Kuswanto et al, 2016; O'Donovan et al, 2009; Reininghaus et al, 2013; Tamminga et al, 2013). Consistent with this model, the findings of minimal CA1 volume trajectory differences between the non-remitters who remained persistently symptomatic at a subthreshold level and those who progressed to full-blown psychosis suggest that the CA1 volume changes may be most closely linked, in a dimensional manner, to the emergence of psychotic symptoms, rather than to the diagnosis of schizophrenia per se.

\section{Possible Underlying Cellular Mechanisms}

The progressive reduction of CA1 volume in individuals with persistent subthreshold symptoms could be related to changes in neuronal, glial, synaptic or vascular cytoarchitecture, or density over time (Ho et al, 2013). Post-mortem studies attempting to identify cytoarchitectural changes in the hippocampus in schizophrenia have generally found no reductions in the number of pyramidal neurons (Heckers et al, 1991; Konradi et al, 2011; Walker et al, 2002) but some evidence for decreased neuronal size and dendritic density throughout the hippocampus (Arnold et al, 1995; Benes et al, 1991, 2001; Rosoklija et al, 2000; Zaidel et al, 1997). Among the hippocampal subfields, CA1 has the largest number of $\gamma$ aminobutyric acid interneurons, which are decreased in density in patients with schizophrenia (Konradi et al, 2011). Based on this finding and related work, it has been proposed that the balance between excitation and inhibition is disrupted in CA1, as a result of either blockage of the $\mathrm{N}$ methyl-D-aspartate receptors on interneurons or a reduction in the number of interneurons, leading to disinhibition of the CA1 pyramidal cells (Heckers and Konradi, 2015; Lisman et al, 2010). Extending this hypothesis, we suggest that this excitatory-inhibitory imbalance leads to alterations in synaptic architecture and density in CA1, and an overall thinning of the CA1 apical neuropil.

\section{Limitations and Future Directions}

These findings should be viewed in light of several limitations of the study. First, the sample size of the converters is modest. The rates of conversion in the present sample cohort (14\%) are lower than the initial UHR cohorts ( 30-40\%) (Cannon et al, 2008; Yung et al, 2003), although they are similar to recent at-risk cohorts across multiple worldwide sites (Cannon et al, 2016; Carrion et al, 2016; Ruhrmann et al, 2010); indeed, rates of conversion have been steadily declining over the past decade to $\sim 7-15 \%$ (FusarPoli et al, 2015; Hartmann et al, 2016).

Second, the present findings of progressive CA1 volume decline in the converters was significant only in the right hemisphere; this appears inconsistent with prior work in prodromal subjects, which detected metabolic and shape changes primarily in the left CA1 (Schobel et al, 2013). However, it is notable that both the prior and current study 
observed similar trends in the contralateral hemisphere. In light of this and the modest sample sizes of these studies, differences in image acquisition and analysis methods, as well as the lack of evidence for any strong laterality of hippocampal abnormalities in schizophrenia (Okada et al, 2016), we suggest that the changes in CA1 volume over time associated with psychotic symptoms are not limited to one hemisphere.

Third, the internal boundaries of the hippocampal subfields are not visible at $1 \mathrm{~mm}$ resolution, thus the automated segmentation method relies heavily on prior knowledge (ie, the probabilistic ex vivo atlas). While segmentations on $1 \mathrm{~mm}$ data cannot delineate the subfields as accurately as higher resolution scans, it has been shown, on several large publicly available data sets of different age groups and disorders, that the segmentations on $1 \mathrm{~mm}$ scans are highly reliable (Whelan et al, 2016), even across different MRI scanner platforms and field strengths (Whelan et al, 2016). Also, on large data sets with the same resolution as our data, it has been demonstrated that the segmentations based on local changes within the hippocampal subfield(s) can discriminate between diseased and healthy states with higher accuracy than the whole hippocampus (Iglesias et al, 2015).

Future larger-scale, prospective studies of UHR subjects could assess the value of including a CA1 volume measure in a multidomain index, which may include proinflammatory cytokines in the plasma (Cannon et al, 2015), negative symptoms (Piskulic et al, 2012), cognitive abilities (Lin et al, 2013), and white matter markers (Bloemen et al, 2010), designed to predict risk and transition to full psychosis.

\section{FUNDING AND DISCLOSURE}

The authors declare no conflict of interest.

\section{ACKNOWLEDGMENTS}

This work was supported by the National Medical Research Council Translational and Clinical Research Flagship Programme (grant number NMRC/TCR/003/2008), Biomedical Research Council, Singapore (grant number BMRC 04/1/ 36/19/372 to JZ), National Medical Research Council, Singapore (grant number CBRG/0088/2015 to JZ), and National Medical Research Council Centre Grant Programme (grant number NMRC/CG/004/2013 to NFH). JL is further supported by the National Healthcare Group Clinician Scientist Career Scheme. JEI is supported by a Marie Sklodowska-Curie fellowship (Project No. 654911 'THALAMODEL') and by the Spanish Ministry of Economy and Competitiveness (reference TEC2014-51882P). DJH is supported by the National Institute of Mental Health (grant number RO1MH095904). The funding bodies had no roles in the design of the study, collection and analysis of data, and decision to publish.

\section{REFERENCES}

Addington J, Heinssen R (2012). Prediction and prevention of psychosis in youth at clinical high risk. Annu Rev Clin Psychol 8: 269-289.
Adriano F, Caltagirone C, Spalletta G (2012). Hippocampal volume reduction in first-episode and chronic schizophrenia: a review and meta-analysis. Neuroscientist 18: 180-200.

Arnold SE, Franz BR, Gur RC, Gur RE, Shapiro RM, Moberg PJ et al (1995). Smaller neuron size in schizophrenia in hippocampal subfields that mediate cortical-hippocampal interactions. Am J Psychiatry 152: 738-748.

Benes FM, Sorensen I, Bird ED (1991). Reduced neuronal size in posterior hippocampus of schizophrenic patients. Schizophr Bull 17: 597-608.

Benes FM, Todtenkopf MS, Kostoulakos P (2001). GluR5,6,7 subunit immunoreactivity on apical pyramidal cell dendrites in hippocampus of schizophrenics and manic depressives. Hippocampus 11: 482-491.

Bloemen OJ, de Koning MB, Schmitz N, Nieman DH, Becker HE, de Haan L et al (2010). White-matter markers for psychosis in a prospective ultra-high-risk cohort. Psychol Med 40: 1297-1304.

Bois C, Levita L, Ripp I, Owens DC, Johnstone EC, Whalley HC et al (2014). Longitudinal changes in hippocampal volume in the Edinburgh High Risk Study of Schizophrenia. Schizophr Res 173: 146-151.

Buehlmann E, Berger GE, Aston J, Gschwandtner U, Pflueger MO, Borgwardt SJ et al (2010). Hippocampus abnormalities in at risk mental states for psychosis? A cross-sectional high resolution region of interest magnetic resonance imaging study. J Psychiatr Res 44: 447-453.

Burwell RD, Agster KL (2009). Anatomy of the Hippocampus and the Declarative Memory System, 1st edn. Elsevier: Amsterdam, The Netherlands.

Cannon TD, Cadenhead K, Cornblatt B, Woods SW, Addington J, Walker E et al (2008). Prediction of psychosis in youth at high clinical risk: a multisite longitudinal study in North America. Arch Gen Psychiatry 65: 28-37.

Cannon TD, Chung Y, He G, Sun D, Jacobson A, van Erp TG et al (2015). Progressive reduction in cortical thickness as psychosis develops: a multisite longitudinal neuroimaging study of youth at elevated clinical risk. Biol Psychiatry 77: 147-157.

Cannon TD, Yu C, Addington J, Bearden CE, Cadenhead KS, Cornblatt BA et al (2016). An individualized risk calculator for research in prodromal psychosis. Am J Psychiatry 173: 980-988.

Carpenter WT (2014). Attenuated psychosis syndrome: need for debate on a new disorder. Psychopathology 47: 287-291.

Carrion RE, Cornblatt BA, Burton CZ, Tso IF, Auther AM, Adelsheim $S$ et al (2016). Personalized prediction of psychosis: external validation of the NAPLS-2 psychosis risk calculator with the EDIPPP Project. Am J Psychiatry 173: 989-996.

Cha J, Greenberg T, Song I, Blair Simpson H, Posner J, MujicaParodi LR (2016). Abnormal hippocampal structure and function in clinical anxiety and comorbid depression. Hippocampus 26: 545-553.

Craddock N, O'Donovan MC, Owen MJ (2009). Psychosis genetics: modeling the relationship between schizophrenia, bipolar disorder, and mixed (or 'schizoaffective') psychoses. Schizophr Bull 35: 482-490.

Cuthbert BN, Insel TR (2010). Toward new approaches to psychotic disorders: the NIMH Research Domain Criteria project. Schizophr Bull 36: 1061-1062.

Dean DJ, Orr JM, Bernard JA, Gupta T, Pelletier-Baldelli A, Carol EE et al (2015). Hippocampal shape abnormalities predict symptom progression in neuroleptic-free youth at ultrahigh risk for psychosis. Schizophr Bull 42: 161-169.

First MB, Spitzer RL, Gibbon M, Williams JBW (2002a). Structured Clinical Interview for DSM-IV-TR Axis I Disorders, Research Version, Patient Edition With Psychotic Screen Biometrics Research. New York State Psychiatric Institute: New York, NY.

First MB, Spitzer RL, Gibbon M, Williams JBW (2002b). Structured Clinical Interview for DSM-IV-TR Axis I Disorders, Research 
Version, Non-patient Edition Biometrics Research. New York State Psychiatric Institute: New York, NY.

Fischl B, Salat DH, Busa E, Albert M, Dieterich M, Haselgrove C et al (2002). Whole brain segmentation: automated labeling of neuroanatomical structures in the human brain. Neuron 33: 341-355.

Fischl B, Salat DH, van der Kouwe AJ, Makris N, Segonne F, Quinn BT et al (2004). Sequence-independent segmentation of magnetic resonance images. NeuroImage 23(Suppl 1): S69-S84.

Fox J, Weisberg S (eds) (2011). An R Companion to Applied Regression. Sage: Thousand Oaks, CA.

Francis AN, Seidman LJ, Tandon N, Shenton ME, Thermenos HW, Mesholam-Gately RI et al (2013). Reduced subicular subdivisions of the hippocampal formation and verbal declarative memory impairments in young relatives at risk for schizophrenia. Schizophr Res 151: 154-157.

Fusar-Poli P, Borgwardt S, Crescini A, Deste G, Kempton MJ, Lawrie $S$ et al (2011). Neuroanatomy of vulnerability to psychosis: a voxel-based meta-analysis. Neurosci Biobehav Rev 35: $1175-1185$.

Fusar-Poli P, Cappucciati M, Rutigliano G, Schultze-Lutter F, Bonoldi I, Borgwardt S et al (2015). At risk or not at risk? A metaanalysis of the prognostic accuracy of psychometric interviews for psychosis prediction. World Psychiatry 14: 322-332.

Fusar-Poli P, Yung AR, McGorry P, van Os J (2014). Lessons learned from the psychosis high-risk state: towards a general staging model of prodromal intervention. Psychol Med 44: 17-24.

Ganzola R, Maziade M, Duchesne S (2014). Hippocampus and amygdala volumes in children and young adults at high-risk of schizophrenia: research synthesis. Schizophr Res 156: 76-86.

Goldman HH, Skodol AE, Lave TR (1992). Revising axis V for DSM-IV: a review of measures of social functioning. $A m J$ Psychiatry 149: 1148-1156.

Haijma SV, Van Haren N, Cahn W, Koolschijn PC, Hulshoff Pol HE, Kahn RS (2013). Brain volumes in schizophrenia: a metaanalysis in over 18000 subjects. Schizophr Bull 39: 1129-1138.

Hartmann JA, Yuen HP, McGorry PD, Yung AR, Lin A, Wood SJ et al (2016). Declining transition rates to psychotic disorder in ultra-high risk clients: investigation of a dilution effect. Schizophr Res 170: 130-136.

Heckers S, Heinsen H, Geiger B, Beckmann H (1991). Hippocampal neuron number in schizophrenia. A stereological study. Arch Gen Psychiatry 48: 1002-1008.

Heckers S, Konradi C (2015). GABAergic mechanisms of hippocampal hyperactivity in schizophrenia. Schizophr Res 167: 4-11.

Heckers S, Konradi C (2010). Hippocampal pathology in schizophrenia. Curr Top Behav Neurosci 4: 529-553.

Hill SK, Reilly JL, Keefe RS, Gold JM, Bishop JR, Gershon ES et al (2013). Neuropsychological impairments in schizophrenia and psychotic bipolar disorder: findings from the Bipolar-Schizophrenia Network on Intermediate Phenotypes (B-SNIP)study. Am J Psychiatry 170: 1275-1284.

Ho NF, Iglesias J, Sum M, Kuswanto C, Sitoh Y, de Souza J et al (2016). Progression from selective to general involvement of hippocampal subfields in schizophrenia. Mol Psychiatry 42: 161-169.

Ho NF, Hooker JM, Sahay A, Holt DJ, Roffman JL (2013). In vivo imaging of adult human hippocampal neurogenesis: progress, pitfalls and promise. Mol Psychiatry 18: 404-416.

Hothorn T, Bretz F, Westfall P (2008). Simultaneous Inference in general parametric models. Biometr J 50: 346-363.

Hurlemann R, Jessen F, Wagner M, Frommann I, Ruhrmann S, Brockhaus A et al (2008). Interrelated neuropsychological and anatomical evidence of hippocampal pathology in the at-risk mental state. Psychol Med 38: 843-851.

Iglesias JE, Augustinack JC, Nguyen K, Player CM, Player A, Wright M et al (2015). A computational atlas of the hippocampal formation using ex vivo, ultra-high resolution MRI: application to adaptive segmentation of in vivo MRI. NeuroImage 115: 117-137.

Kapur S, Phillips AG, Insel TR (2012). Why has it taken so long for biological psychiatry to develop clinical tests and what to do about it? Mol Psychiatry 17: 1174-1179.

Klauser P, Zhou J, Lim JK, Poh JS, Zheng H, Tng HY et al (2015). Lack of evidence for regional brain volume or cortical thickness abnormalities in youths at clinical high risk for psychosis: findings from the Longitudinal Youth at Risk Study. Schizophr Bull 41: 1285-1293.

Konradi C, Yang CK, Zimmerman EI, Lohmann KM, Gresch P, Pantazopoulos $\mathrm{H}$ et al (2011). Hippocampal interneurons are abnormal in schizophrenia. Schizophr Res 131: 165-173.

Kuswanto C, Chin R, Sum MY, Sengupta S, Fagiolini A, McIntyre RS et al (2016). Shared and divergent neurocognitive impairments in adult patients with schizophrenia and bipolar disorder: Whither the evidence? Neurosci Biobehav Rev 61: 66-89.

Lee J, Rekhi G, Mitter N, Bong YL, Kraus MS, Lam M et al (2013). The Longitudinal Youth at Risk Study (LYRIKS) - an Asian UHR perspective. Schizophr Res 151: 279-283.

Lin A, Yung AR, Nelson B, Brewer WJ, Riley R, Simmons M et al (2013). Neurocognitive predictors of transition to psychosis: medium- to long-term findings from a sample at ultra-high risk for psychosis. Psychol Med 43: 2349-2360.

Lisman JE, Pi HJ, Zhang Y, Otmakhova NA (2010). A thalamohippocampal-ventral tegmental area loop may produce the positive feedback that underlies the psychotic break in schizophrenia. Biol Psychiatry 68: 17-24.

McDonald C, Marshall N, Sham PC, Bullmore ET, Schulze K, Chapple B et al (2006). Regional brain morphometry in patients with schizophrenia or bipolar disorder and their unaffected relatives. Am J Psychiatry 163: 478-487.

O'Donovan MC, Craddock NJ, Owen MJ (2009). Genetics of psychosis; insights from views across the genome. Hum Genet 126: $3-12$.

O'Driscoll GA, Florencio PS, Gagnon D, Wolff AV, Benkelfat C, Mikula L et al (2001). Amygdala-hippocampal volume and verbal memory in first-degree relatives of schizophrenic patients. Psychiatry Res 107: 75-85.

Okada N, Fukunaga M, Yamashita F, Koshiyama D, Yamamori H, Ohi $\mathrm{K}$ et al (2016). Abnormal asymmetries in subcortical brain volume in schizophrenia. Mol Psychiatry 21: 1460-1466.

Ongur D, Cullen TJ, Wolf DH, Rohan M, Barreira P, Zalesak M et al (2006). The neural basis of relational memory deficits in schizophrenia. Arch Gen Psychiatry 63: 356-365.

Piskulic D, Addington J, Cadenhead KS, Cannon TD, Cornblatt BA, Heinssen $\mathrm{R}$ et al (2012). Negative symptoms in individuals at clinical high risk of psychosis. Psychiatry Res 196: 220-224.

Reininghaus U, Priebe S, Bentall RP (2013). Testing the psychopathology of psychosis: evidence for a general psychosis dimension. Schizophr Bull 39: 884-895.

Reuter M, Schmansky NJ, Rosas HD, Fischl B (2012). Withinsubject template estimation for unbiased longitudinal image analysis. NeuroImage 61: 1402-1418.

Rosoklija G, Toomayan G, Ellis SP, Keilp J, Mann JJ, Latov N et al (2000). Structural abnormalities of subicular dendrites in subjects with schizophrenia and mood disorders: preliminary findings. Arch Gen Psychiatry 57: 349-356.

Ruhrmann S, Schultze-Lutter F, Salokangas RK, Heinimaa M, Linszen D, Dingemans P et al (2010). Prediction of psychosis in adolescents and young adults at high risk: results from the prospective European prediction of psychosis study. Arch Gen Psychiatry 67: 241-251.

Schobel SA, Chaudhury NH, Khan UA, Paniagua B, Styner MA, Asllani I et al (2013). Imaging patients with psychosis and a mouse model establishes a spreading pattern of hippocampal dysfunction and implicates glutamate as a driver. Neuron 78: 81-93. 
Seidman LJ, Faraone SV, Goldstein JM, Kremen WS, Horton NJ, Makris N et al (2002). Left hippocampal volume as a vulnerability indicator for schizophrenia: a magnetic resonance imaging morphometric study of nonpsychotic first-degree relatives. Arch Gen Psychiatry 59: 839-849.

Seidman LJ, Rosso IM, Thermenos HW, Makris N, Juelich R, Gabrieli JD et al (2014). Medial temporal lobe default mode functioning and hippocampal structure as vulnerability indicators for schizophrenia: a MRI study of non-psychotic adolescent firstdegree relatives. Schizophr Res 159: 426-434.

Simon AE, Velthorst E, Nieman DH, Linszen D, Umbricht D, de Haan L (2011). Ultra high-risk state for psychosis and nontransition: a systematic review. Schizophr Res 132: 8-17.

Smith MJ, Cobia DJ, Reilly JL, Gilman JM, Roberts AG, Alpert KI et al (2015). Cannabis-related episodic memory deficits and hippocampal morphological differences in healthy individuals and schizophrenia subjects. Hippocampus 25: 1042-1051.

Solowij N, Walterfang M, Lubman DI, Whittle S, Lorenzetti V, Styner $\mathrm{M}$ et al (2013). Alteration to hippocampal shape in cannabis users with and without schizophrenia. Schizophr Res 143: $179-184$.

Talati P, Rane S, Kose S, Blackford JU, Gore J, Donahue MJ et al (2014). Increased hippocampal CA1 cerebral blood volume in schizophrenia. NeuroImage Clin 5: 359-364.

Tamminga CA, Ivleva EI, Keshavan MS, Pearlson GD, Clementz BA, Witte B et al (2013). Clinical phenotypes of psychosis in the Bipolar-Schizophrenia Network on Intermediate Phenotypes (BSNIP). Am J Psychiatry 170: 1263-1274.

The R Core Team (2015). R: A Language and Environment For Statistical Computing. The R Core Team: Vienna, Austria.

Thompson PM, Hayashi KM, Simon SL, Geaga JA, Hong MS, Sui Y et al (2004). Structural abnormalities in the brains of human subjects who use methamphetamine. J Neurosci 24: 6028-6036.

van Erp TG, Hibar DP, Rasmussen JM, Glahn DC, Pearlson GD, Andreassen OA et al (2015). Subcortical brain volume abnormalities in 2028 individuals with schizophrenia and 2540 healthy controls via the ENIGMA consortium. Mol Psychiatry 21: 547-553.

Velakoulis D, Wood SJ, Wong MT, McGorry PD, Yung A, Phillips L et al (2006). Hippocampal and amygdala volumes according to psychosis stage and diagnosis: a magnetic resonance imaging study of chronic schizophrenia, first-episode psychosis, and ultrahigh-risk individuals. Arch Gen Psychiatry 63: 139-149.

Walker MA, Highley JR, Esiri MM, McDonald B, Roberts HC, Evans SP et al (2002). Estimated neuronal populations and volumes of the hippocampus and its subfields in schizophrenia. Am J Psychiatry 159: 821-828.

Wang C, Ji F, Hong Z, Poh JS, Krishnan R, Lee J et al (2016). Disrupted salience network functional connectivity and white matter microstructure in persons at risk for psychosis: findings from the LYRIKS study. Psychol Med 46: 2771-2783.

Whelan CD, Hibar DP, van Velzen LS, Zannas AS, Carrillo-Roa T, McMahon $\mathrm{K}$ et al (2016). Heritability and reliability of automatically segmented human hippocampal formation subregions. NeuroImage 128: 125-137.

Whittle S, Lichter R, Dennison M, Vijayakumar N, Schwartz O, Byrne ML et al (2014). Structural brain development and depression onset during adolescence: a prospective longitudinal study. Am J Psychiatry 171: 564-571.

Witthaus H, Kaufmann C, Bohner G, Ozgurdal S, Gudlowski Y, Gallinat J et al (2009). Gray matter abnormalities in subjects at ultra-high risk for schizophrenia and first-episode schizophrenic patients compared to healthy controls. Psychiatry Res 173: 163-169.

Witthaus H (2010). Hippocampal subdivision and amygdalar volumes in patients in an at-risk mental state for schizophrenia. J Psychiatry Neurosci 35: 33-40.

Wood SJ, Kennedy D, Phillips LJ, Seal ML, Yucel M, Nelson B et al (2010). Hippocampal pathology in individuals at ultra-high risk for psychosis: a multi-modal magnetic resonance study. NeuroImage 52: 62-68.

Wood SJ, Yucel M, Velakoulis D, Phillips LJ, Yung AR, Brewer W et al (2005). Hippocampal and anterior cingulate morphology in subjects at ultra-high-risk for psychosis: the role of family history of psychotic illness. Schizophr Res 75: 295-301.

Yung AR, Nelson B, Thompson A, Wood SJ (2010). The psychosis threshold in ultra high risk (prodromal) research: is it valid? Schizophr Res 120: 1-6.

Yung AR, Philips LJ, Yuen HP, McGorry P (eds) (2006). Comprehensive Assessment of at Risk Mental State. The PACE Clinic, ORYGEN Research Centre, University of Melbourne: Parkville, VIC, Australia.

Yung AR, Phillips LJ, Yuen HP, Francey SM, McFarlane CA, Hallgren $M$ et al (2003). Psychosis prediction: 12-month follow up of a high-risk ('prodromal') group. Schizophr Res 60: 21-32.

Yung AR, Yuen HP, McGorry PD, Phillips LJ, Kelly D, Dell'Olio M et al (2005). Mapping the onset of psychosis: the Comprehensive Assessment of At-Risk Mental States. Aust NZ J Psychiatry 39: 964-971.

Zaidel DW, Esiri MM, Harrison PJ (1997). Size, shape, and orientation of neurons in the left and right hippocampus: investigation of normal asymmetries and alterations in schizophrenia. Am J Psychiatry 154: 812-818.

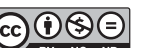

This work is licensed under a Creative Commons Attribution-NonCommercial-NoDerivs 4.0 International License. The images or other third party material in this article are included in the article's Creative Commons license, unless indicated otherwise in the credit line; if the material is not included under the Creative Commons license, users will need to obtain permission from the license holder to reproduce the material. To view a copy of this license, visit http://creativecommons.org/licenses/by-nc-nd/4.0/

(C) The Author(s) 2017

Supplementary Information accompanies the paper on the Neuropsychopharmacology website (http://www.nature.com/npp) 